\title{
What Happened on Restart a Heart Day 2017 in England?
}

Terry P Brown ${ }^{1}$, Gavin D Perkins ${ }^{1}$, Andy Lockey ${ }^{2}$, Jasmeet Soar ${ }^{2}$, Sara Askew ${ }^{3}$, Frank Mersom ${ }^{4}$, Rachael T Fothergill Emma Cox $^{6}$, Sara Black ${ }^{7}$, Jenny Lumley-Holmes ${ }^{8}$, OHCAO collaborators

Purpose of the Study:

Restart a Heart (RSaHD) is an annual European day of action that aims to teach life-saving CPR skills to as many people as possible. In 2017, UK stakeholders collaborated to train at least 195,000 young people (and a few adults) in CPR. The event was organised by a partnership between the Resuscitation Council (UK), British Heart Foundation, British Red Cross, St John Ambulance and all UK ambulance services. In 2018 RSaHD is going global under the oversight of ILCOR (http://www.ilcor.org/wrah/). The aim of this simple study is to report details of the out-of-hospital cardiac arrests (OHCAs) that occurred on RSaHD 2017 in English NHS Ambulance Service Trusts.

\section{Results:}

Table shows the demographics and outcomes of 48 OHCAs attended/treated by ambulance service clinicians. Nine cases were declared deceased on scene; 29 cases were admitted to hospital with ongoing resuscitation, 7 of which had achieved ROSC but subsequently re-arrested whilst being attended by ambulance clinicians. 10 cases were admitted with ROSC.

rom the incidence in the Table, and the mid-year populations for England $(55,619430)$ and UK $(66,040229)$, we estimate that on RSaHD in 2017, there were about 78 cases in England (UK=92), of which about 44 (UK=52) would have received bystander CPR, about 16 (UK=19) cases would have achieved ROSC at hospital handover and $3(U K=4)$ survived to hospital discharge.

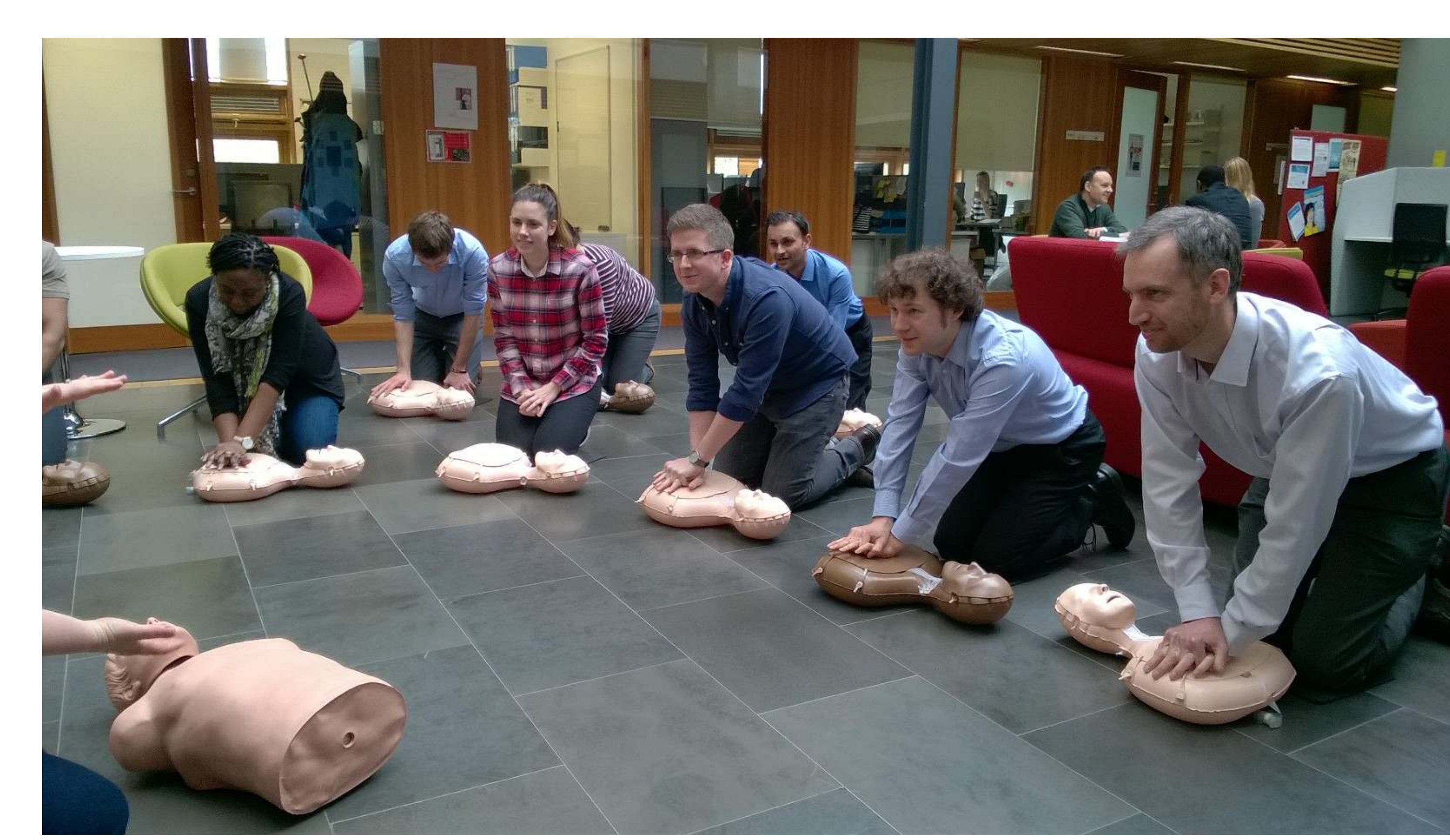

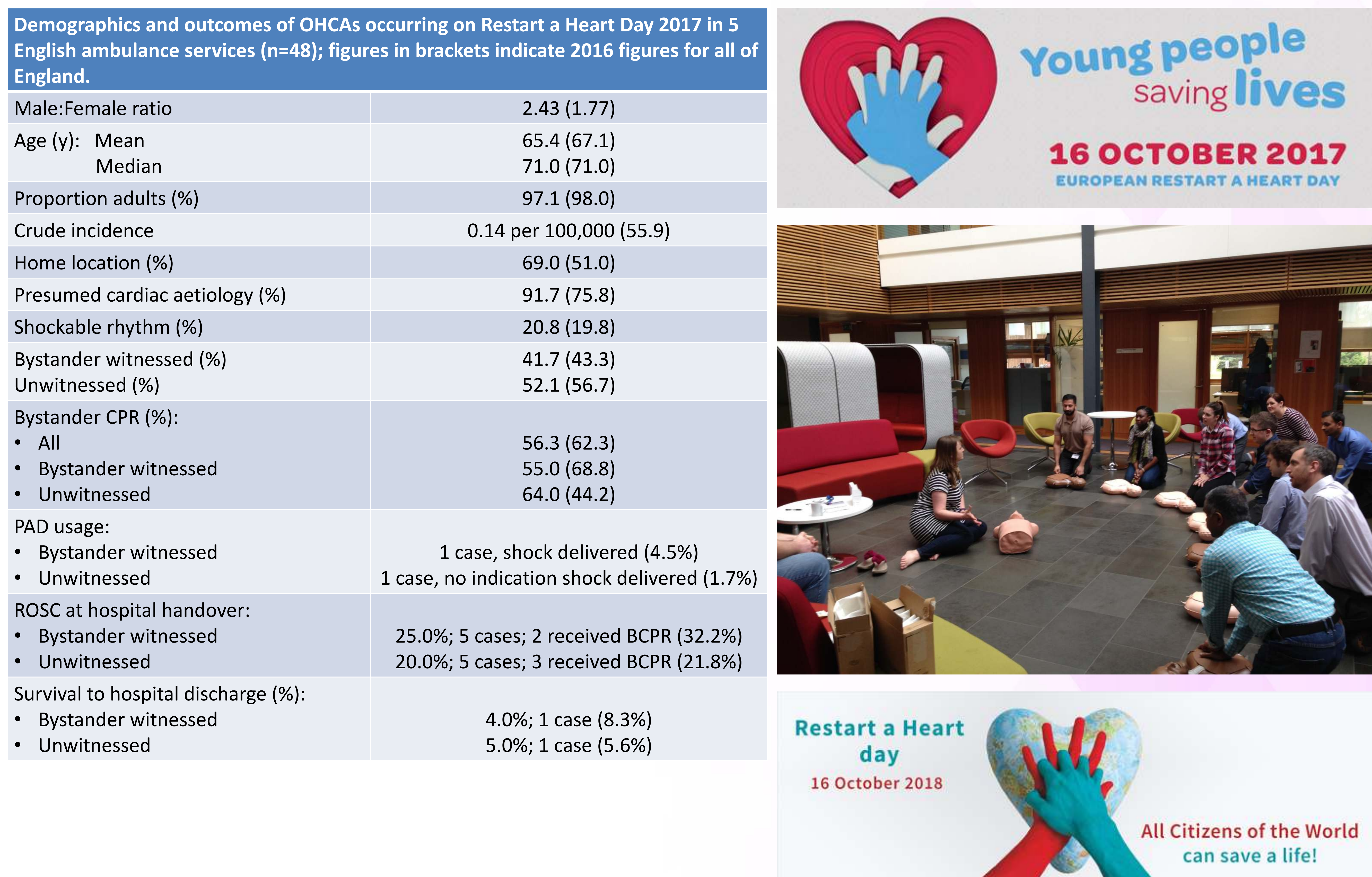

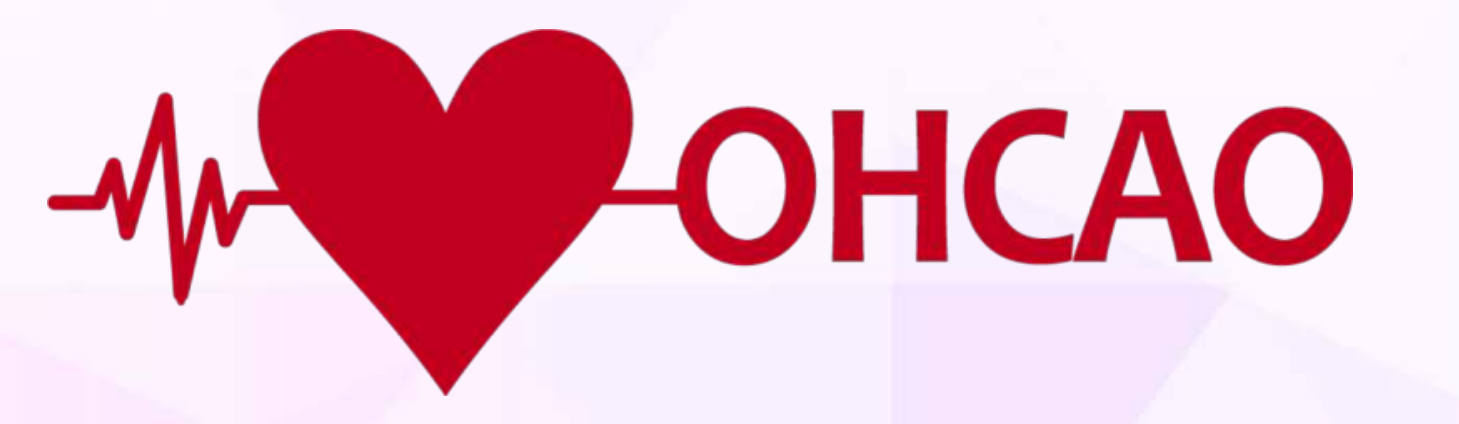

Conclusions:

This crude, 1-day snapshot, analysis estimated that on Restart a Heart Day in 2017 about 75 lives were lost to cardiac arrest in England (88 in UK).

Having the Out-of-Hospital Cardiac Arrest Outcomes registry will enable us to

continually monitor the impact of Restart a Heart Day, and other training initiatives, on outcomes after OHCA in the UK for the foreseeable future.
Details of OHCAs that occurred on $16^{\text {th }}$ October 2017 in 5 English ambulance services were obtained from the $\mathrm{OHCA}$ Outcomes registry at University of Warwick. A descriptive Outcomes registry at Univers
analysis was undertaken. 